\title{
Review of: "Elastic ice microfibers"
}

\author{
Changqing Sun ${ }^{1}$ \\ 1 Nanyang Technological University
}

Potential competing interests: The author(s) declared that no potential competing interests exist.

This breakthrough [1, 2] challenged the limit of experimental techniques and imagination. It is of great importance to the understanding of water ice under multifield perturbation in the following aspects:

1. Electric field promotes the low temperature, fast, and directional growth of quality fibres. The known bulk freezing point $T_{N}=258 \mathrm{~K}$ [3] has been reduced from $35 \mathrm{~K}$. The O:H nonbond energy dictates the $T_{N}$ and the $T_{V}$ for evaporation and the $\mathrm{H}-\mathrm{O}$ energy governs the melting point $T_{m}[4]$. Therefore, electrification shortens and stiffens the $\mathrm{H}-\mathrm{O}$ bond but does the O:H contrastingly.

2. The maximal elastic strain increases from $4.6 \%$ to $10.9 \%$ as the operating temperature decreases from 200 to $120 \mathrm{~K}$ and the feature size is reduced from 4.6 to $4.4 \mathrm{~mm}$. I wonder if the scale size discrimination of the operating temperatures is inclusive. More testing would be wonderful.

3. The bending of the fibre at $220 \mathrm{~K}$ adds two sets of satellites to the Raman peaks of Ice- $\mathrm{h}(220,3110$ $\left.\mathrm{cm}^{-1}\right)$. One set at $\left(158,3225 \mathrm{~cm}^{-1}\right)$ may arise from then tension and the other unmarked at $(270,2900$ $\mathrm{cm}^{-1}$ ) may from compression as compression softens the $\mathrm{H}-\mathrm{O}$ and stiffens the $\mathrm{O}: \mathrm{H}$ while tension does it contrastingly [5].

4. The super rigidity-elasticity transition at the cryotemperature and the microscopic scale evidence the multifield coupling effect (positive and negative pressure, temperature, molecular undercoordination, etc.) on the extraordinary mechanical, optical, and thermal properties of the supersolid state.

5. Discoveries of the super-elasticity of "ice" should add aspects to understanding the performance of the "no man's land" water at 120-200 K and its core-shell or coordination enviroment resolved biphase supersolidity [6].

1. Xu, P., B. Cui, Y. Bu, H. Wang, X. Guo, P. Wang, Y.R. Shen, and L. Tong, Elastic ice microfibers. Science, 2021. 373(6551): p. 187-192.

2. Schulson, E.M., A flexible and springy form of ice. Science, 2021. 373(6551): p. 158-158.

3. Sun, C.Q., X. Zhang, X. Fu, W. Zheng, J.-I. Kuo, Y. Zhou, Z. Shen, and J. Zhou, Density and phononstiffness anomalies of water and ice in the full temperature range. J. Phys. Chem. Lett., 2013. 4: p. 32383244.

4. Sun, C.Q., Water electrification: Principles and applications. Adv. Coll. Interf. Sci., 2020. 282: p. 102188.

5. Li, L., M. Bo, J. Li, Y. Huang, X. Zhang, and C.Q. Sun, Water ice compression: Principles and 
applications. J. Mol. Liq., 2020. 315: p. 113705.

6. Sun, C.Q., Rules essential for water molecular undercoordination. Chin. Phys. B, 2020. 8(8): p. 088203. 